\title{
Efficient Silicon Device Simulation with the Local Iterative Monte Carlo Method
}

\author{
JÜRGEN JAKUMEIT ${ }^{\mathrm{a}, *}$, TORSTEN MIETZNER ${ }^{\mathrm{b}}$ and UMBERTO RAVAIOLI ${ }^{\mathrm{c}, \dagger}$ \\ ${ }^{a}$ Institute for Algorithm and Scientific Computing (SCAI) GMD-German National Research Center for Information \\ Technology Schloß Birlinghoven, 53754 Sankt Augustin, Germany; ${ }^{\mathrm{b}}$ II. Physikalisches Institut, Universität Köln, \\ Zülpicher Str. 77, 50937 Köln, Germany; ${ }^{\mathrm{c}}$ Beckman Institute, University of Illinois at Urbana/Champaign, USA
}

\begin{abstract}
The Local Iterative Monte Carlo technique (LIMO) is used for an effective simulation of hot electron distributions in silicon MOSFETs. This new Monte Carlo approach yields an efficient use of the computational resources due to a different iteration scheme. In addition the necessary computation time can be further reduced by a reuse of the computational expensive MC step simulation results in the iteration process. The later possibility is investigated in detail in this work. Results for short channel MOSFETs demonstrates that correct two-dimensional hot electron distributions can be calculated by LIMO within 1 hour on a standard work station.
\end{abstract}

Keywords: Monte Carlo; Semiconductor devices; Local Iterative Monte Carlo; Short channel SiMOSFETs

\section{INTRODUCTION}

In typical particle simulations applied to device problems it is desirable to simulate regions having widely different carrier concentration with similar resolution of the energy distribution function. Standard Monte Carlo (MC) algorithms [1,2] use variance reduction techniques to limit the statistical error in regions of low carrier density [3-5]. We introduced an alternative approach based on a Local Iterative Monte Carlo (LIMO) algorithm. The evolution of the energy distribution function from an initial distribution to steady state is calculated by an iterative application of short local MC steps for test particles that represent the local density $[6,7]$. In contrast to standard MC algorithms the charge density represented by the test particle is proportional to the local density and the number of particles simulated is the same for low and high density regions. This leads to a more efficient use of computational resources and uniform statistical error. Meanwhile a two-dimensional version of the LIMO technique for the simulation of silicon devices, based on the full band structure, was implemented and tested.

In this contribution we focus on the possibility for a further computation time reduction by tabulating and using more than once the results

*Tel.: + 492214 14-2746, Fax: -2181, e-mail: jakumeit@gmd.de

${ }^{\dagger}$ Corresponding author. Tel.: 1 2172445765, Fax: 1 2172444333, e-mail: ravaioli@uiuc.edu 
from the local MC steps in the overall iteration process. Since the MC step simulations need most of the computation time, using each drift information in more than one iteration can significantly reduce the computational expenses. Our investigation revealed that especially at high dimensional simulations this feature can significantly reduce the computation time. The following chapter describes the LIMO technique. Chapter 3 discusses the reuse of drift information from MC-steps and demonstrates the efficient calculation of hot electron distributions in a $75 \mathrm{~nm}$ MOSFET using LIMO as post-processor.

\section{LIMO ALGORITHM}

The LIMO technique is based on a phase space discretization and consists of two parts, the short local MC-steps applied to each phase space cell and the iteration process, which combines the MC-steps to a complete simulation. For the MCsteps a test carrier is placed randomly inside the phase space cell. The drift and scattering process of this carrier are simulated in the same way as in a conventional MC-simulation to a final momentum $\mathbf{k}_{\text {final }}$ and position $\mathbf{r}_{\text {final }}$. In this way each phase space cell is connected to a final position in phase space reflecting a possible carrier trajectory in this region. The final positions can be collected in a table for a reuse during the iteration process. The more MC-steps are collected the better is the statistical representation of the underlying physics. The iteration process starts from any initial carrier distribution. In each iteration a part of the carrier density at each phase space cell is moved to the cells surrounding the final position given by the MC-step result. In this way the initial distribution is changed until steady state is reached. The steady state distribution is a solution of the Boltzmann transport equation [7].

The parallelization of the LIMO is simple since the local MC-steps need no information from the iteration process. Therefore the algorithm can be effectively parallelized by a client/server strategy. As many clients as possible calculate the short MC carrier trajectories and create the corresponding tables. The server uses these table for the iterative electron density redistribution. An almost linear speed up can be achieved when the number of nodes is smaller then the number of MC-steps, calculated for each phase space cell [6].

The LIMO algorithm used in this work is tailored for a fast calculation of the hot electron distribution in Si-MOSFETs. Not the complete momentum but only the energy of the test particles is memorized in the tables. The MC simulation of short electron trajectories uses the full band structure and includes scattering due to phonon absorption and emission, impact ionization and ionzed impurities as well as surface scattering. The algorithm is used as post-processor. The spacial electron distribution and electric field profile for the MC-step simulation are taken from hydrodynamic simulations or standard MC simulations. As an example Figure 1 compares results from the LIMO algorithm with those from MOCA2D [8] and Damocles [9], the two-dimensional device simulator from the University of Illinois and IBM, respectively. The energy distribution of electrons along the channel is plotted for the important region at the crossover from channel to drain.

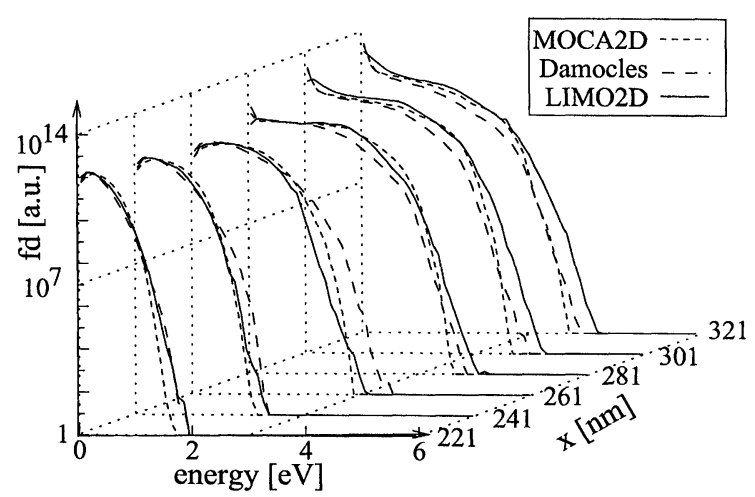

FIGURE 1 Energy distribution of electrons along the channel for a $75 \mathrm{~nm} \operatorname{MOSFET}\left(V_{\mathrm{d}}=V_{\mathrm{g}}=3 \mathrm{~V}\right)$. Results from LIMO, MOCA and Damocles are compared. These x-dependent distribution was obtained from the $2 \mathrm{D}$ ones by integration over the depth of the structure. 


\section{REUSE OF DRIFT INFORMATION}

For the application as fast post-processor for nonself-consistent calculations the possibility to use the MC-step results more then once in the iteration process is an important feature of the LIMO algorithm. While the iteration process needs 30 or more iterations to reach steady state our computer experiments show that a significant lower number of MC-steps simulations per phase space cell is necessary for a sufficient representation of the underlying physics. The number of MC-steps is sufficient when the result of the LIMO algorithm, i.e., the energy distribution of electrons, does not improve with increasing MC-step number. Figure 2 shows the energy distributions of electrons in a $75 \mathrm{~nm}$ Si-MOSFET along the channel for 2D LIMO calculations with 1,3,10 and 30 MC-steps per phase space cell. The displayed one-dimensional distribution is obtained from the twodimensional one by integration over the depth of the structure. The results for 3,10 and $30 \mathrm{MC}$ steps can hardly be distinguished, indicating that 3 MC-step calculations per phase space cell are enough to represent the underlying physics.

A more rigorous analysis of the efficiency of the reuse of drift information is presented in Figure 3. Here the fluctuations in LIMO results are plotted as a function of the MC-step number for a bulk,

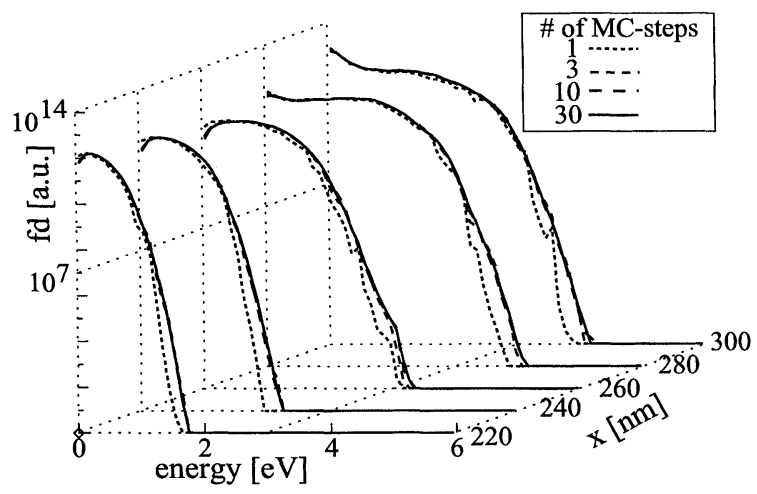

FIGURE 2 Energy distribution of electrons along the channel for a $75 \mathrm{~nm} \operatorname{MOSFET}\left(V_{\mathrm{d}}=V_{\mathrm{g}}=3 \mathrm{~V}\right)$ obtained using different numbers of MC-steps per phase space cell.

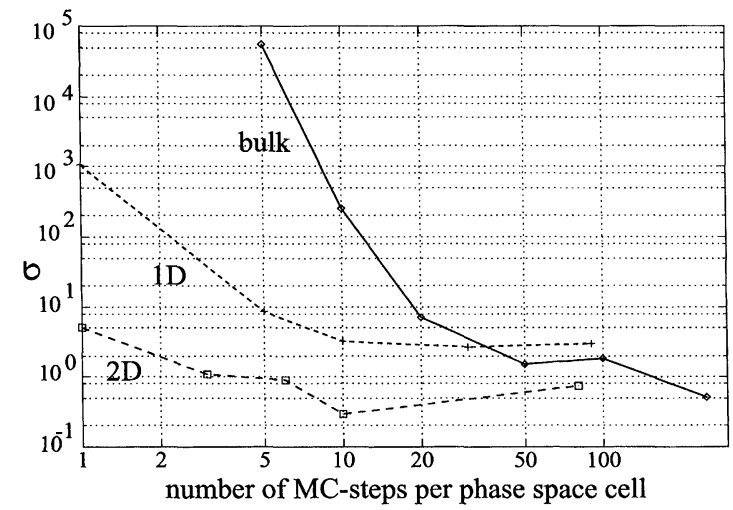

FIGURE 3 Fluctuations $\sigma$ in the gate current as function of the number of MC-steps for bulk, 1D and 2D LIMO simulations.

1D and 2D LIMO algorithm. Fluctuations of the gate current $I_{\mathrm{g}}$ are used as measure for the fluctuations in the energy distributions of electrons. $I_{\mathrm{g}}$ was calculated include tunneling, image lowering of the barrier, and a probability of being backscattered due to collisions in the oxide [8]. It can be seen as a measure for the fluctuations in the distribution around $3.2 \mathrm{eV}$ at the transition from channel to drain. For the $1 \mathrm{D}$ and $2 \mathrm{D}$ results the $75 \mathrm{~nm}$ MOSFET discussed above was used as test structure. For the bulk case the electron concentration above $3.2 \mathrm{eV}$ was used instead of $I_{\mathrm{g}}$ and a field of $200 \mathrm{kV} / \mathrm{cm}$ was used in the simulation. The fluctuations in $I_{\mathrm{g}}$ are measured by performing 10 simulations with identical input and calculating the standard deviation $\sigma$ in the gate currents $I_{\mathrm{i}}$ of the individual runs $(i=1, \ldots, N=10): \sigma=$ $\sqrt{\sum_{i}\left(I_{i}-I_{\mathrm{avg}}\right)^{2}} /\left(\sqrt{N} \cdot I_{\mathrm{avg}}\right)$. The three curves for bulk, 1D and 2D simulations show the same behavior. The fluctuations saturate with increasing number of MC-steps per phase space cell to the value obtained in the ideal case, when the MC-step results are not reused at all (right most points in Fig. 3). But the curve for the $2 \mathrm{D}$ case saturates already with $3 \mathrm{MC}$-steps, while about $30 \mathrm{MC}$-steps must be calculated in the bulk case to reach a sufficient representation of the underlying physics. This dimensionality dependence can be understood if one considers, that the physics in a region 
is not only represented by the MC results of the corresponding phase space cell but also by the results of the surrounding cells. The carrier density taken from the start cell is distributed among the cells surrounding the final energy $\mathbf{E}_{\text {final }}$ and position $\mathbf{r}_{\text {final }}$ and these cells are therefore connected. As a consequence less MC-steps per phase space cell are necessary to describe a region with sufficient statistics with increasing dimensionality due to the larger number of neighboring cells. Therefore, the reuse of drift information becomes increasingly efficient with increasing dimension of the simulation and can partly compensate the increasing amount of computation time due to the increasing grid size.

As an example for the application of LIMO as post-processor for drift-diffusion or hydrodynamic simulations Figure 4 compares the electron temperature distribution in the $75 \mathrm{~nm}$ MOSFET calculated by LIMO based on an electric field and carrier density profile calculated
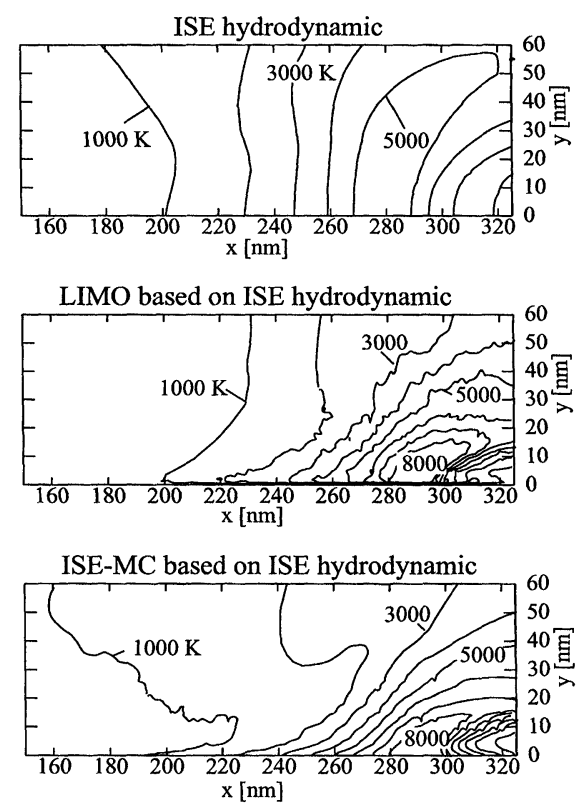

FIGURE 4 2D electron average energy distribution in a $75 \mathrm{~nm}$ MOSFET $\left(V_{\mathrm{d}}=V_{\mathrm{g}}=3 \mathrm{~V}\right)$, calculated with a hydrodynamic model (ISE-TCAD DESSIS) (upper), the LIMO technique (middle) and the ISE MC program (ISE-DEGAS) (lower). using the ISE-TCAD hydrodynamic model with the hydrodynamic findings and ISE-TCAD MC results. Less than 1 hour was need for the LIMO calculation of a energy distribution of electrons, which is comparable to the MC result.

\section{CONCLUSION}

The possibility for a further computation time reduction in the LIMO technique by tabulating and using more than once the results from the local MC steps in the overall iteration process was investigated. Our results reveal that especially in higher dimensional simulations the computational expensive drift information can be very efficiently used by the LIMO technique. A two-dimensional (2D) simulation of a Si-MOSFET is possible in less then an hour on a modern PC. This makes LIMO an interesting post-processor to driftdiffusion or hydrodynamic simulations for the calculation of hot electron effects.

\section{Acknowledgments}

This work was partially supported by the Semiconductor Research Corporation, contract 99-NJ726 , by the Distributed Center for Advanced Electronics Simulation(DesCArtES) through $\mathrm{Na}-$ tional Science Foundation grant ECS 98-02730, and the Deutsche Forschungsgemeinschaft GK Scientific Computing. The authors would like to thank the Integrated System Laboratory at the ETH Zürich for making available the ISE-TCAD simulator.

\section{References}

[1] Fawcett, W., Boardman, A. D. and Swain, S. (1970). J. Chem. Solids, 31, 1963-1990.

[2] Jacoboni, C. and Reggiani, R. (1983). Rev. Mod. Phys., 55, 645-705.

[3] Fischetti, M. V. and Laux, S. E. (1988). Phys. Rev. B, 38, 9721.

[4] Pacelli, A. and Ravaioli, U. (1997). Solid-State Electron., 41, 599-605.

[5] Wordelman, J., Kwan, T. J. T. and Snell, C. M. (1998). IEEE Trans. Computer Aided Design, 17, 1230-1234. 
[6] Jakumeit, J., Sontowski, T. and Ravaioli, U., Proceedings of IWCE-6, pp. 92-95, Osaka, Japan, October 19-21, 1998.

[7] Jakumeit, J. and Ravaioli, U., Semiconductor transport simulation with the Local Iterative Monte Carlo Technique, submitted to IEEE Trans. ED.
[8] Duncan, A., Ravaioli, U. and Jakumeit, J. (1998). IEEE Trans. ED, 45, 867-876.

[9] Laux, S. E. and Fischetti, M. V. (1991). In: Monte Carlo Device Simulation: Full Band and Beyond, Ed. Hess, K. Kluwer, pp. 1-26. 

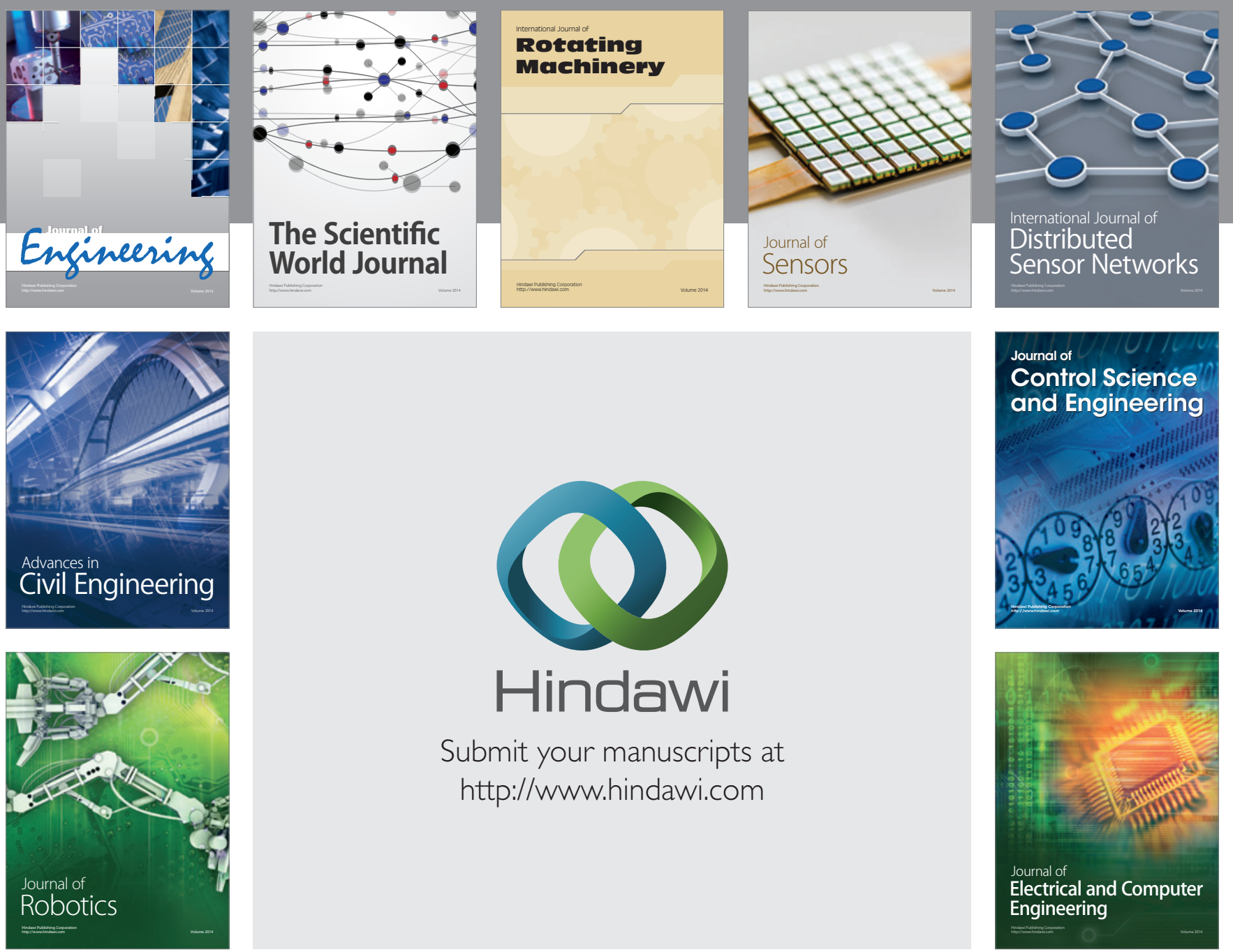

Submit your manuscripts at

http://www.hindawi.com
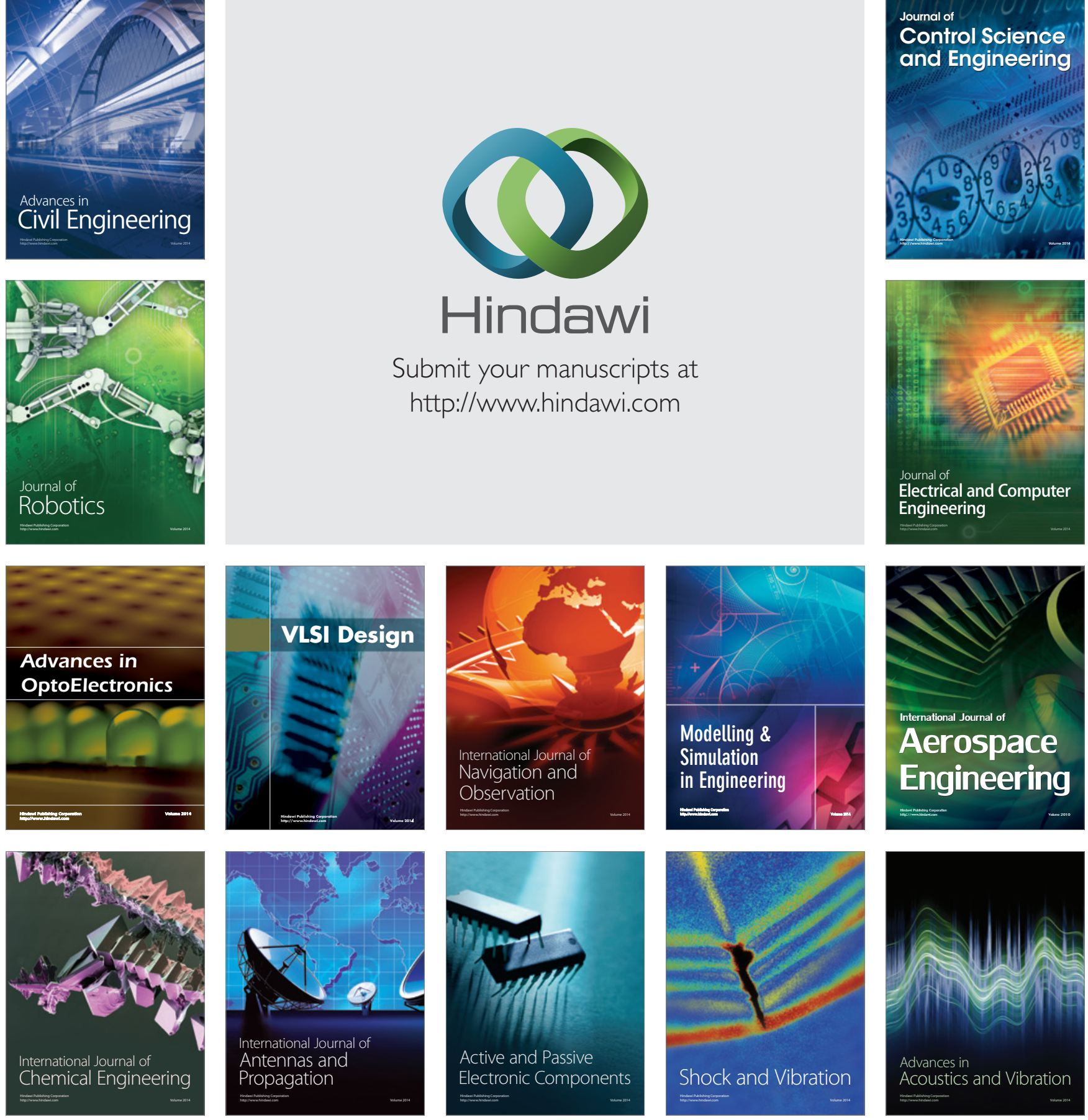